\title{
Syndrome De Raynaud Paraneoplasique : A Propos D'un Cas. Paraneoplastic Raynaud Syndroma: About One Case.
}

\author{
${ }^{1}$ Bouzaiene $\mathrm{H},{ }^{2}$ Chemlali M, ${ }^{3}$ Triki A, ${ }^{4}$ Ben Hassouna J, ${ }^{5}$ Slimane M, \\ ${ }^{6}$ Chargui R, ${ }^{7}$ Laamouri B, ${ }^{8}$ Ayadi MA, ${ }^{9}$ Hechiche M, ${ }^{10}$ Gamoudi A, ${ }^{11}$ Dhiab T, \\ ${ }^{12}$ Rahal K. \\ Service de Chirurgie Carcinologique, Institut Salah Azaï,, Tunis, Tunisie
}

\begin{abstract}
The Raynaud syndroma is a frequent reason of consultation in rheumatology. Most often, the Raynaud syndroma is without underlying disease. However, in rare cases, it can be associated to a cancer, so that it is called paraneoplastic Raynaud syndroma. The breast cancer is not knowen as giving paraneoplastic syndroma. We report one case of breast cancer associated to a Raynaud syndroma .
\end{abstract}

Key-words: Breast cancer - paraneoplastic Raynaud syndroma - mammectomy

\section{Introduction}

Le syndrome de Raynaud est un trouble de la circulation sanguine se manifestant par un engourdissement ou des douleurs des extrémités (le plus souvent les mains). Dans les cas les plus sévères, une nécrose des doigts peut survenir. Ce syndrome peut être secondaire à d'autres maladies, et dans ce cas il est appelé syndrome de Raynaud secondaire. Une cause sous-jacente n'est retrouvée que dans 10 à $20 \%$ des cas [1]. Quand aucune cause n'est retrouvée, et ce le cas le plus fréquent, il est dit primitif.

Il est parfois le premier signe révélateur de la maladie, notamment dans les sclérodermies [2]. Ailleurs il peut rentrer dans le cadre d'un syndrome paranéoplasique, généralement secondaire à un cancer du poumon ou du rein. L'association à un cancer du sein est exceptionnelle.

\section{Observation}

Il s'agit d'une patiente âgée de 61 ans, hypertendue, qui a consulté pour une lésion croûteuse mamelonaire gauche évoluant depuis trois mois.

L'examen clinique a trouvé une patiente en bon état général. A l'examen mammaire, il y avait un aspect de croûte sur le mamelon gauche évoquant une maladie de Paget du mamelon (Fig.1). La palpation mammaire était normale et les aires ganglionnaires axillaires et sus-claviculaires étaient libres. Par ailleurs, l'examen des deux mains a trouvé une nécrose des pulpes des $2^{\text {ème }}, 4^{\text {ème }}$ et $5^{\text {ème }}$ doigts droits et $2^{\text {ème }}, 3^{\text {ème }}$ doigts gauches épargnant les pouces(Fig.2). Le reste de l'examen somatique était sans particularités.

La mammographie a montré des microcalcifications suspectes diffuses à tout le sein gauche à maximum au niveau du quadrant supéro-externe gauche classées ACR5. L'échographie mammaire n'a pas été faite. La biopsie a conclu à un carcinome in situ de haut grade.

Dans le cadre de l'exploration de la nécrose digitale, l'écho-doppler était normale.

Devant ces constatations anatomo-cliniques et radiologiques, le diagnostic de syndrome de Raynaud paranéoplasique secondaire à un carcinome in situ du sein gauche a été évoqué. La décision thérapeutique était d'effectuer une mammectomie gauche avec prélèvement ganglionnaire axillaire homolatéral.

L'étude anatomopathologique définitive de la pièce de mammectomie a conclu à un carcinome intra-canalaire prédominant avec composante canalaire infiltrante grade II modifié et une maladie de Paget du mamelon du sein gauche. Les ganglions prélevés étaient réactionnels.

L'évolution de la maladie était marquée par l'amélioration spectaculaire des lésions cutanées digitales avec atténuation remarquable du syndrome de Raynaud juste après la chirurgie. Ce qui a permis de retenir définitivement le diagnostic de syndrome de Raynaud paranéoplasique.

\section{Discussion}

En 1862, Maurice Raynaud décrit un phénomène paroxystique évoluant en trois phases : ischémique avec un blanchissement des extrémités par vasoconstriction des artères digitales, des artérioles précapillaires et des shunts artérioveineux cutanés (nécessaire pour poser le diagnostic), hyperhémique inconstante au cours de laquelle les extrémités deviennent rouges, puis un retour à la normale [3] . L'atteinte touche la plupart des doigts commençant parfois par un nombre limité de doigts et épargnant volontiers les pouces. Le nez, les oreilles, la langue peuvent être également touchés. 
Le diagnostic positif est clinique; la capillaroscopie péri-unguéale et les examens immunologiques sont essentiellement une aide au diagnostic étiologique.

La compréhension des mécanismes pathogéniques est longtemps restée une énigme. Plusieurs mécanismes favorisant la vasoconstriction ont été évoqués: l'activité des $\alpha 2$-adrénorécepteurs, l'augmentation de la production d'endothéline 1[4], la probable intervention de l'angiotensine II, de la sérotonine, une augmentation de l'activité de la tyrosine kinase par les cellules endothéliales [6,7].

Le déficit des fibres nerveuses innervant les capillaires entraîne une diminution de production de substances vasodilatatrices tels des neuropeptides (calcitonin gene related peptide, substance P, neurokinine A, neuropeptide $\mathrm{Y}$, peptide vaso-intestinal), agissant par le biais de la production de monoxyde d'azote. Le rôle des modifications intravasculaires est moins clair. En effet, dans le syndrome de Raynaud paranéoplasique, tous ces mécanismes interfèrent ensemble. Dans la maladie de Raynaud, il existe des troubles surtout fonctionnels[5]. Le phénomène de Raynaud est dit primitif si aucune pathologie sous-jacente n'est retrouvée et dans ce cas il est dit également maladie de Raynaud. L'existence d'antécédents familiaux est en faveur d'une atteinte primitive particulièrement chez les sujets jeunes [8]. Chez les femmes, la répartition entre maladie de Raynaud et syndrome de Raynaud secondaire est respectivement de $85 \%$ et $15 \%$ alors que chez les hommes, elle est de 50 et $50 \%$.

Sur le plan pratique, les critères diagnostiques de la maladie de Raynaud sont le déclenchement par le froid et/ou le stress, l'atteinte symétrique et bilatérale, l'absence de nécrose, l'absence de cause sous-jacente, l'absence de syndrome inflammatoire, la négativité des facteurs antinucléaires et la capillaroscopie normale.

Dans le cas de notre patiente, malgré la faible positivité des facteurs anti-nucléaires et le fait que la capillaroscopie n'a pas été faite, le diagnostic de syndrome de Raynaud secondaire paranéoplasique était fortement évoqué et définitivement retenu devant la disparition des lésions cutanées après le traitement chirurgical du cancer du sein et l'amélioration spectaculaire de la symptomatologie avant même le début de tout traitement adjuvant.

Pour le volet thérapeutique, quand le phénomène de Raynaud est primitif, les inhibiteurs calciques sont le traitement de référence. Au cours des dernières années, des thérapeutiques vasodilatatrices puissantes : prostacycline et dérivés, les antagonistes des récepteurs de l'endothéline, les inhibiteurs des phosphodiestérase sont apparues et indiquées notamment dans les formes sévères et résistantes

Quand le phénomène de Raynaud est secondaire, comme c'est le cas de notre patiente, le traitement est celui de la maladie sous-jacente.

\section{Conclusion}

Le syndrome de Raynaud paranéoplasique est rare, cependant il faut y penser devant toute association avec une pathologie cancéreuse même si cette pathologie n'est pas réputée pourvoyeuse de syndrome paranéoplasique. D'autant plus que le traitement est celui du cancer sous-jacent.

\section{References}

[1] Block JA, Sequeira W. Raynaud's phenomenon. Lancet 2001;357:2042-8.

[2] Wigley FM. Raynaud's phenomenon. N Engl J Med 2002;347:1001-8.

[3] O'Keefe ST, Tsapatsaris NP, Beetham WP. Increased prevalence of migraine and chest pain in patients with primary Raynaud's disease. Ann Intern Med 1992;116:985-9.

[4] Carpentier PH. Définition et épidémiologie des acrosyndromes vasculaires. Rev Prat 1998;46:1641-6.

[5] Planchon B, Pistorius MA, Beurrier P, de Faucal P. Primary's Raynaud's phenomenon. Age of onset and pathogenesis in a prospective study of 424 patients. Angiology 1994:45:677-86.

[6] Olsen N, Nielsen SL. Prevalence of primary Raynaud phenomena in young females. Scand J Clin Lab Invest 1978;37:761-4

[7] Furspan PB, Chatterjee S, Mayes MD, Freedman RR. Cooling-induced contraction and protein tyrosine kinase activity of isolated arterioles in secondary Raynaud's phenomenon. Rheumatology 2005;44:488-94.

[8] Furspan PB, Chatterjee S, Freedman RR. Increased tyrosine phosphorylation mediates the cooling-induced contraction and increased vascular reactivity of Raynaud's disease. Arthritis Rheum 2004;50:1578-85. 


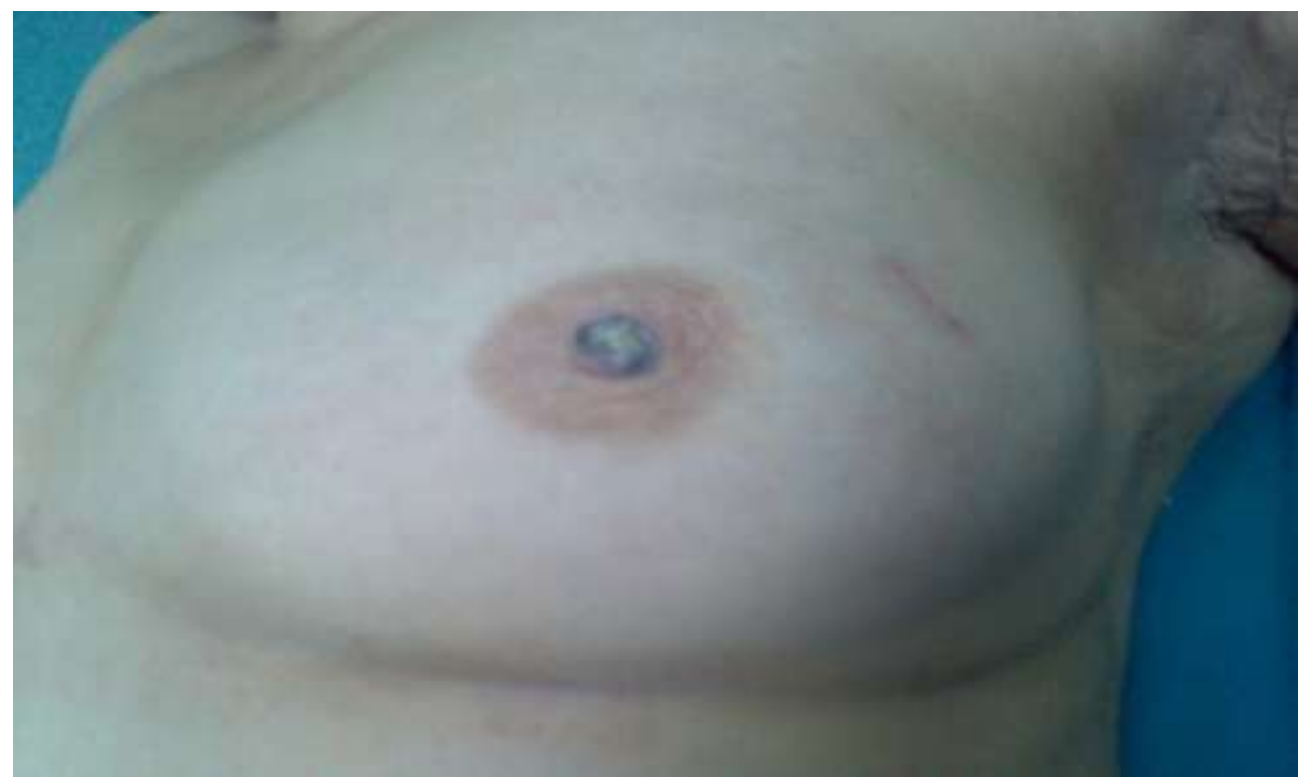

Fig 1: Aspet crouteux du mamelon

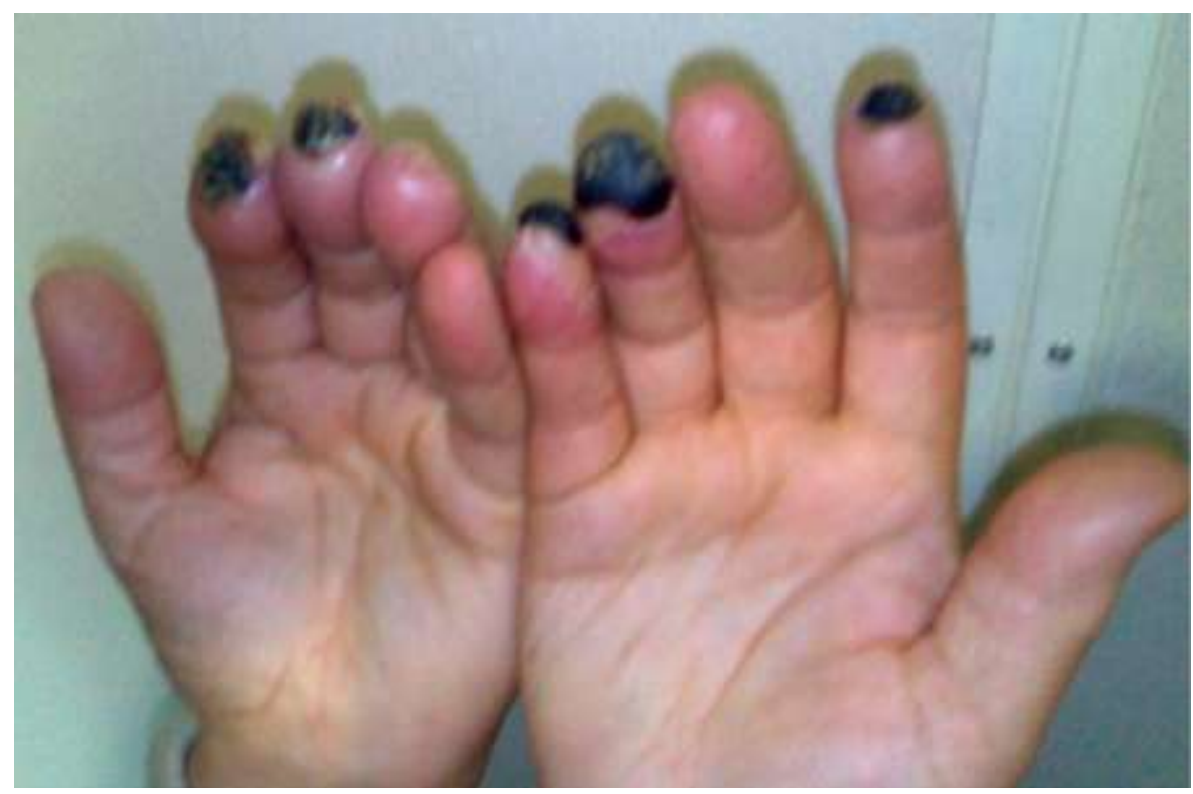

Fig 2: Nécrose de la pulpe des doigts 